\title{
Spatial smoothing with uniform circular arrays
}

\author{
K MAHESWARA REDDY ${ }^{*}$ and V U REDDY \\ Electrical Communication Engineering Department, Indian Institute of \\ Science, Bangalore, India \\ "Present address: Centre for Aeronautical Systems Studies and Analysis, De- \\ fence Research and Development Organisation, New Tippasandra, Bangalore \\ 560075 , India \\ e-mail: mahesh@cassa.ernet.in; vur@ece.iisc.ernet.in
}

\begin{abstract}
In this paper, we extend and analyse spatial smoothing with uniform circular arrays (UCA's). In particular, we study the performance of the Root-MUSIC with smoothing in the presence of correlated sources, finite data perturbations and errors in transformed steering vector that arise due to some approximations made to enable the extension of the Root-MUSIC and smoothing to UCA. Expressions are derived for the asymptotic performance of the Root-MUSIC with smoothing applied to the transformed UCA data. An attempt has been made to bring out the impact of both forward and forward-backward smoothing. Computer simulations are provided to demonstrate the usefulness of the analysis.
\end{abstract}

Keywords. Uniform circular arrays; phase modes; direction of arrival estimation; spatial smoothing.

\section{Introduction}

Uniform circular arrays (UCA's) are commonly employed when $360^{\circ}$ coverage is required in the plane of the array. Circular arrays are non-uniform linear arrays, and hence, the rooting techniques and preprocessing schemes like spatial smoothing cannot be directly applied to these arrays. Tewfik \& Hong (1992) showed that it is possible to extend the Root-MUSIC to UCA using the phase mode excitation concept. Mathews \& Zoltowski (1994) proposed real beamspace MUSIC to UCA (UCA-RB-MUSIC) which yields reduced computation and better resolution. They also studied the direction of arrival (DOA) estimation performance of the UCA-RB-MUSIC.

While extending the rooting techniques to UCA, all the authors assumed that some of the terms in the transformed steering vector of UCA, where the transformation is performed with phase mode excitation vectors, are negligible when the circumferential spacing between the elements is less than half wavelength. These approximations cause errors in the 
DOA estimates obtained with the Root-MUSIC even when the number of snapshots tends to infinity, and we analyse the effect of these errors in this paper. We also extend spatial smoothing to UCA and analyse the effect of smoothing in the presence of correlated sources and finite data perturbations. We discuss the impact of both forward and forward-backward smoothing.

In $\S 2$, we provide a brief background. We propose in $\S 3$ forward spatial smoothing highlighting the assumptions made for extending the Root-MUSIC and spatial smoothing to UCA and the errors associated with these assumptions. In $\S 4$, we analyze the performance of the Root-MUSIC with forward and forward-backward smoothing applied to the transformed UCA data. We present the results of computer simulations in $\S 6$ and conclude the paper in $\$ 7$.

\section{Background}

Consider a UCA with $L$ identical and omni-directional sensors. Let $r$ be the radius of the array and $d$ be the circumferential spacing between the elements. Let $\theta$ denote the angle (azimuth angle) measured in the plane containing the elements. We assume for simplicity that the sources are in the same plane as the UCA. The steering vector of the UCA w.r.t. the centre of the array can then be expressed as 1

$$
\mathbf{a}_{c}(\theta)=\left[e^{j \xi \cos \theta}, e^{j \xi \cos (\theta-2 \pi / L)}, \ldots, e^{j \xi \cos (\theta-2 \pi(L-1) / L)}\right]^{\mathrm{T}}
$$

where $\xi=2 \pi r / \lambda, \lambda$ is the wavelength and $(.)^{\mathrm{T}}$ represents the transpose of $($.$) .$

Consider the phase mode excitation of the UCA. The weight vector that excites the array with $m$ th phase mode is given by (Davies 1983) $\mathbf{w}_{m}^{\mathrm{H}}=\left(j^{-|m|} / L\right)\left[1, e^{j 2 \pi m / L}, \ldots\right.$, $\left.e^{j 2 \pi m(L-1) / L}\right]$. The array pattern for the $m$ th phase mode can be shown to be given by (Davies 1983; Mathews \& Zoltowski 1994)

$$
\begin{aligned}
f_{m}(\theta)= & \mathbf{w}_{m}^{\mathrm{H}} \mathbf{a}_{c}(\theta)=J_{|m|}(\xi) e^{j m \theta} \\
& +j^{-|m|} \sum_{q=1}^{\infty}\left[j^{g} J_{g}(\xi) e^{-j g \theta}+j^{h} J_{h}(\xi) e^{j h \theta}\right] ; \quad-\mathcal{D} \leq m \leq \mathcal{D},
\end{aligned}
$$

where $\mathcal{D}$ is the maximum number of phase modes given by (Davies 1983) $\mathcal{D} \simeq\lfloor 2 \pi r / \lambda\rfloor$, $J_{m}(\xi)$ is the Bessel function of the first kind of order $m, h=L q+m, g=L q-m,(.)^{\mathrm{H}}$ represents the complex conjugate transpose of (.) and $\lfloor x\rfloor$ denotes the largest integer less than or equal to $x$. The first term in (2), the principal term, becomes dominant if $d$ is less than $0.5 \lambda$. In our analysis, we consider $d<0.5 \lambda$ and assume the second term of (2) to be negligible.

The normalised transformation matrix $\mathbf{F}$ to excite the array patterns corresponding to $(2 \mathcal{D}+1)$ phase modes is given by $\mathbf{F}=\sqrt{\mathcal{L}}\left[\mathbf{w}_{-\mathcal{D}}, \ldots, \mathbf{w}_{0}, \ldots, \mathbf{w}_{\mathcal{D}}\right]$. Using this transformation, we express

$$
\mathbf{a}_{t}(\theta)=\mathbf{F}^{\mathrm{H}} \mathbf{a}_{c}(\theta)=\mathbf{J}_{\xi} \mathbf{a}(\theta)+\Delta \mathbf{a}(\theta)
$$

\footnotetext{
${ }^{1}$ If the sources are at some elevation angle, say $\phi$, the steering vector can still be expressed by (1) with $\xi$ defined as $\xi=\frac{2 \pi r \sin \phi}{\lambda}$ where $\phi$ is measured w.r.t. the perpendicular to the array.
} 
where

$$
\begin{aligned}
& \mathbf{J}_{\xi}=\sqrt{L} \operatorname{diag}\left[J_{\mathcal{D}}(\xi), \ldots, J_{1}(\xi) . J_{0}(\xi), J_{1}(\xi), \ldots, J_{\mathcal{D}}(\xi)\right] \\
& \mathbf{a}(\theta)=\left[e^{-j \mathcal{D} \theta}, e^{-j(\mathcal{D}-1) \theta}, \ldots, 1, \ldots, e^{j(\mathcal{D}-1) \theta}, e^{j \mathcal{D} \theta}\right]^{\mathrm{T}}
\end{aligned}
$$

and $\Delta \mathbf{a}(\theta)$ is the contribution due to the second term in (2). Note that the vector $\mathbf{a}(\theta)$ has a structure similar to that of the steering vector of a uniform linear array (ULA). This suggests that we can extend the spatial smoothing to UCA provided the term $\Delta \mathbf{a}(\theta)$ is negligible. We treat $\Delta \mathbf{a}(\theta)$ as the error in the transformed steering vector, caused due to approximation.

\section{Forward spatial smoothing with UCA}

Assume that $M$ sources are impinging on the UCA and the DOA's of these sources are $\theta_{1}, \theta_{2}, \ldots, \theta_{M}$. If we assume that the signal and noise are uncorrelated and noise is spatially white with variance $\sigma^{2}$, then the covariance matrix at the output of UCA can be expressed as

$$
\mathbf{R}_{c}=\mathbf{A}_{c} \mathbf{S A}_{c}^{\mathrm{H}}+\sigma^{2} \mathbf{I}
$$

where $\mathbf{S}$ is the signal covariance matrix, $\mathbf{I}$ is an identity matrix and $\mathbf{A}_{c}$ is the matrix of direction vectors of the UCA. From (5) and (3), the covariance matrix that we obtain after applying the transformation $\mathbf{F}$ can be shown to be

$$
\mathbf{R}^{t}=\mathbf{F}^{\mathrm{H}} \mathbf{R}_{c} \mathbf{F}=\mathbf{J}_{\xi} \mathbf{A} \mathbf{S} \mathbf{A}^{\mathrm{H}} \mathbf{J}_{\xi}+\sigma^{2} \mathbf{I}+\Delta \mathbf{R},
$$

where $\mathbf{A}=\left[\mathbf{a}\left(\theta_{1}\right), \ldots, \mathbf{a}\left(\theta_{M}\right)\right]$ and $\Delta \mathbf{R}=\Delta \mathbf{A} \mathbf{S} \mathbf{A}^{\mathrm{H}} \mathbf{J}_{\xi}+\mathbf{J}_{\xi} \mathbf{A} \mathbf{S} \Delta \mathbf{A}^{\mathrm{H}}+\Delta \mathbf{A} \mathbf{S} \Delta \mathbf{A}^{\mathrm{H}}$ with $\Delta \mathbf{A}=\left[\Delta \mathbf{a}\left(\theta_{1}\right), \ldots, \Delta \mathbf{a}\left(\theta_{M}\right)\right]$. Note that the size of $\mathbf{R}^{t}$ is $(2 \mathcal{D}+1) \times(2 \mathcal{D}+1)$, and hence, the number of sources $(M)$ should be less than $(2 \mathcal{D}+1)$ for any MUSIC-type algorithm to be applied to $\mathbf{R}^{t}$.

Spatial smoothing is a preprocessing scheme originally proposed for ULA to alleviate the ill effects of correlation. This scheme can be extended to UCA by applying the transformation $\mathbf{J}_{\xi}^{-1}$ to the covariance matrix $\mathbf{R}^{t}$ provided the term $\Delta \mathbf{R}$ is negligible. If the sources are in the same plane as the UCA or if all the sources are at the same but known elevation angle, then $\mathbf{J}_{\xi}^{-1}$ is a known matrix.

Let $K$ be the number of virtual subarrays (since the subarrays are not physically available). Then, the forward smoothed covariance matrix $\mathbf{R}_{f}^{t}$ is given by

$$
\mathbf{R}_{f}^{t}=\frac{1}{K} \sum_{l=1}^{K} \mathbf{Z}_{l}^{\mathrm{T}} \mathbf{J}_{\xi}^{-1} \mathbf{R}^{t} \mathbf{J}_{\xi}^{-1} \mathbf{Z}_{l}
$$

where $\mathbf{Z}_{l}=\left[\mathbf{e}_{l}, \mathbf{e}_{l+1}, \ldots, \mathbf{e}_{l+L_{o}-1}\right]$ with $\mathbf{e}_{l}$ denoting the $l$ th column of the identity matrix of size $2 \mathcal{D}+1, L_{o}$ is the virtual subarray dimension with $L_{o}=2 \mathcal{D}-K+2$. It may be noted from (7) and (6) that the noise part of $\mathbf{R}_{f}^{t}$ is diagonal but not of the form $\sigma^{2} \mathbf{I}$. For convenience, we use prewhitening before applying the Root-MUSIC (assuming once again $\Delta \mathbf{R}$ to be negligible). The forward smoothed covariance matrix after prewhitening is given by

$$
\left(\mathbf{R}_{f}^{t}\right)_{w}=\mathbf{R}_{n w} \mathbf{R}_{f}^{t} \mathbf{R}_{n w}^{\mathrm{H}}
$$


where the prewhitening matrix $\mathbf{R}_{n w}=\left[\frac{1}{K} \sum_{l=1}^{K} \mathbf{Z}_{l}^{\mathrm{T}} \mathbf{J}_{\xi}^{-1} \mathbf{J}_{\xi}^{-1} \mathbf{Z}_{l}\right]^{-1 / 2}$. If $K$ is greater than or equal to the number of coherent signals present, then the forward spatial smoothing builds up the rank of the smoothed signal covariance matrix and makes it nonsingular (Shan et al 1985).

Using the structure of $\mathbf{a}(\theta)$ as given in (4), it can be shown (Reddy et al 1987) that the effective correlation coefficient $\left(\rho_{f}\right)$ between the sources after forward smoothing, in the case of UCA, is given by (assuming $\Delta \mathbf{R}$ to be negligible)

$$
\left|\rho_{f}\right|=\left|\rho \frac{\sin \left(K\left(\theta_{i}-\theta_{j}\right) / 2\right)}{K \sin \left(\left(\theta_{i}-\theta_{j}\right) / 2\right)}\right|,
$$

where $\theta_{i}$ and $\theta_{j}$ are the DOA's of the $i$ th and $j$ th sources respectively, and $\rho$ is the correlation coefficient between these sources before smoothing. Note from (9) that $\rho_{f}$ is not dependent on the individual directions of the sources, but is dependent only on their angular separation. If this angular separation is $90^{\circ}$, then $\rho_{f}$ becomes zero for $K=4$. On the other hand, if the separation is $180^{\circ}$, then two subarrays $(K=2)$ are enough to force $\rho_{f}$ to zero. Note also from (9) that $\rho_{f}$ is independent of the spacing between the elements provided $d$ is much less than $\lambda / 2$ (making $\Delta \mathbf{a}(\theta)$, and hence, $\Delta \mathbf{R}$ to be negligible). In contrast, when the forward spatial smoothing is applied to ULA, the effective correlation between the sources is dependent on the individual directions of the sources and also on the spacing between the elements.

\section{Performance of the Root-MUSIC with smoothing}

In this section, we analyze the performance of the Root-MUSIC with forward and forwardbackward smoothing applied to the transformed UCA data.

\subsection{Forward spatial smoothing}

Consider the smoothed covariance matrix after prewhitening (see (8)). Combining this with (6) and (7), we obtain

$$
\begin{aligned}
\left(\mathbf{R}_{f}^{t}\right)_{w}= & \frac{1}{K} \sum_{l=1}^{K} \mathbf{R}_{n w} \mathbf{Z}_{l}^{\mathrm{T}} \mathbf{A S A} \mathbf{A}^{\mathrm{H}} \mathbf{Z}_{l} \mathbf{R}_{n w}^{\mathrm{H}}+\sigma^{2} \mathbf{I} \\
& +\frac{1}{\bar{K}} \sum_{l=1}^{K} \mathbf{R}_{n w} \mathbf{Z}_{l}^{\mathrm{T}} \mathbf{J}_{\xi}^{-1} \Delta \mathbf{R J}_{\xi}^{-1} \mathbf{Z}_{l} \mathbf{R}_{n w}^{\mathrm{H}} \\
= & \mathbf{R}_{F}+\Delta \mathbf{R}_{F},
\end{aligned}
$$

where

$$
\begin{aligned}
\mathbf{R}_{\mathbf{F}} & \equiv \frac{1}{K} \sum_{l=1}^{K} \mathbf{R}_{n w} \mathbf{Z}_{l}^{\mathrm{T}} \mathbf{A} \mathbf{S} \mathbf{A}^{\mathrm{H}} \mathbf{Z}_{l} \mathbf{R}_{n w}^{\mathrm{H}}+\sigma^{2} \mathbf{I} \\
& =\mathbf{R}_{n w} \mathbf{A}_{f} \mathbf{S}_{f} \mathbf{A}_{f}^{\mathrm{H}} \mathbf{R}_{n w}^{\mathrm{H}}+\sigma^{2} \mathbf{I}
\end{aligned}
$$


and

$$
\begin{aligned}
\Delta \mathbf{R}_{\mathbf{F}} & \equiv \frac{1}{K} \sum_{l=1}^{K} \mathbf{R}_{n w} \mathbf{Z}_{l}^{\mathrm{T}} \mathbf{J}_{\xi}^{-1} \Delta \mathbf{R} \mathbf{J}_{\xi}^{-1} \mathbf{Z}_{l} \mathbf{R}_{n w}^{\mathrm{H}} \\
& =\frac{1}{K} \sum_{l=1}^{K} \mathbf{R}_{n w} \mathbf{Z}_{l}^{\mathrm{T}}\left[\mathbf{J}_{\xi}^{-1} \Delta \mathbf{A} \mathbf{S A}^{\mathrm{H}}+\mathbf{A} \mathbf{S} \Delta \mathbf{A}^{\mathrm{H}} \mathbf{J}_{\xi}^{-1}\right] \mathbf{Z}_{l} \mathbf{R}_{n w}^{\mathrm{H}} .
\end{aligned}
$$

$\mathbf{S}_{f}$ is the smoothed signal covariance matrix and $\mathbf{A}_{f}$ is the virtual subarray direction matrix. In writing the RHS of (12), we assumed $\Delta \mathbf{A}$ to be small and neglected the terms containing more than one $\Delta \mathbf{A}$. Note that $\mathbf{R}_{\mathbf{F}}$ is the smoothed covariance matrix that we would get if $\Delta \mathbf{a}(\theta)$ (see (3)) is zero. In practice, however, this term may be small but non-zero, thereby resulting in errors in the DOA estimates when we apply the Root-MUSIC to $\left(\mathbf{R}_{f}^{t}\right)_{w}$. We now analyse the effect of this term (i.e., $\Delta \mathbf{R}_{\mathbf{F}}$ ) and that due to finite data perturbations on the DOA estimates.

Let $\left(\hat{\mathbf{R}}_{f}^{t}\right)_{w}$ denote the estimated covariance matrix from finite number of snapshots. This can be expressed as

$$
\left(\hat{\mathbf{R}}_{f}^{t}\right)_{w}=\left(\mathbf{R}_{f}^{t}\right)_{w}+\Delta \mathbf{R}_{\mathbf{p}}=\mathbf{R}_{\mathbf{F}}+\Delta \mathbf{R}_{\mathbf{F}}+\Delta \mathbf{R}_{\mathbf{p}}
$$

where $\Delta \mathbf{R}_{\mathbf{p}}$ represents the perturbation due to finite data. Note that $\Delta \mathbf{R}_{\mathbf{p}}$ is random while $\Delta \mathbf{R}_{\mathbf{F}}$ is deterministic. If we assume that the noise at the output of the sensors is complex circularly Gaussian distributed, then the mean square error (MSE) in $i$ th DOA estimate, due to both the finite data perturbations and the error due to approximation (i.e. due to $\Delta \mathbf{R}_{\mathbf{F}}$ ), can be shown to be (Rao \& Hari 1990)

$$
\begin{aligned}
E\left[\Delta \theta_{i}^{2}\right]_{f}= & \frac{\Gamma_{\alpha \alpha \beta \beta}+\operatorname{Re}\left(\Gamma_{\alpha \beta \alpha \beta}\right)+2\left[\operatorname{Re}\left(\alpha^{\mathrm{H}} \Delta \mathbf{R}_{\mathbf{F}} \beta\right)\right]^{2}}{2\left[\mathbf{v}_{f_{1}}^{\mathrm{H}}\left(\theta_{i}\right) \mathbf{R}_{n w}^{\mathrm{H}} \mathbf{P}_{n} \mathbf{R}_{n w} \mathbf{v}_{f_{1}}\left(\theta_{i}\right)\right]^{2}} \\
\Gamma_{\alpha \alpha \beta \beta}= & \frac{1}{N K^{2}} \sum_{p=1}^{K} \sum_{q=1}^{K} \alpha^{\mathrm{H}} \mathbf{R}_{p q} \alpha \beta^{\mathrm{H}} \mathbf{R}_{q p} \beta ; \\
\Gamma_{\alpha \beta \alpha \beta}= & \frac{1}{N K^{2}} \sum_{p=1}^{K} \sum_{q=1}^{K} \alpha^{\mathrm{H}} \mathbf{R}_{p q} \beta \alpha^{\mathrm{H}} \mathbf{R}_{q p} \beta . \\
& \alpha=\mathbf{P}_{n} \mathbf{R}_{n w} \mathbf{v}_{f_{1}}\left(\theta_{i}\right) ; \quad \beta=\left(\mathbf{R}_{\mathbf{F}}\right)_{s}^{\#} \mathbf{R}_{n w} \mathbf{v}_{f}\left(\theta_{i}\right) ; \\
\left(\mathbf{R}_{\mathbf{F}}\right)_{s}= & \mathbf{R}_{n w} \mathbf{A}_{f} \mathbf{S}_{f} \mathbf{A}_{f}^{\mathrm{H}} \mathbf{R}_{n w}^{\mathrm{H}},
\end{aligned}
$$

where $\mathbf{R}_{p q}=\mathbf{R}_{q p}^{\mathrm{H}}=\mathbf{E}\left[\mathbf{y}_{p}(t) \mathbf{y}_{q}^{\mathrm{H}}(t)\right], \mathbf{y}_{p}(t)$ is the output vector obtained from the $p$ th virtual subarray after prewhitening, $N$ is the number of snapshots, $\mathbf{P}_{n}$ is the projection matrix onto the noise subspace of $\mathbf{R}_{\mathbf{F}}, \mathbf{v}_{f_{1}}(\theta)$ is the derivative of $\mathbf{v}_{f}(\theta)$ w.r.t. $\theta$ with $\mathbf{v}_{f}(\theta)$ denoting the normalised virtual subarray steering vector, $\operatorname{Re}($.$) represents the real part of$ (.), (.) $)^{\#}$ denotes pseudo inverse of (.) and $E($.$) denotes the expectation of (.).$

When $d$ is much less than $\lambda / 2, \Delta \mathbf{R}_{\mathrm{F}}$ is very small and error in the DOA estimate is mainly because of the noise perturbations due to finite data. This error is given by

$$
E\left[\Delta \theta_{i}^{2}\right]_{f}=\frac{\Gamma_{\alpha \alpha \beta \beta}+\operatorname{Re}\left(\Gamma_{\alpha \beta \alpha \beta}\right)}{2\left[\mathbf{v}_{f_{1}}^{\mathrm{H}}\left(\theta_{i}\right) \mathbf{R}_{n w}^{\mathrm{H}} \mathbf{P}_{n} \mathbf{R}_{n w} \mathbf{v}_{f_{1}}\left(\theta_{i}\right)\right]^{2}} .
$$


Reddy \& Reddy (1996a) showed that the smoothing reduces the noise perturbations due to finite data in addition to reducing the correlation among the impinging sources.

As the number of snapshots tends to infinity, the MSE in the DOA estimate is mainly because of the error due to the approximation (cf. (3)). This error, which we refer to as the asymptotic error in the DOA estimate, is deterministic and given by

$$
E\left[\Delta \theta_{i}^{2}\right]_{f}=\Delta \theta_{i}^{2}=\frac{\left[\operatorname{Re}\left(\alpha^{\mathrm{H}} \Delta \mathbf{R}_{\mathbf{F}} \beta\right)\right]^{2}}{\left[\mathbf{v}_{f_{1}}^{\mathrm{H}}\left(\theta_{i}\right) \mathbf{R}_{n w}^{\mathrm{H}} \mathbf{P}_{n} \mathbf{R}_{n w} \mathbf{v}_{f_{1}}\left(\theta_{i}\right)\right]^{2}}
$$

Note that this error increases as $d$ tends to $\lambda / 2$ since $\Delta \mathbf{A}$ becomes larger with increasing values of $d$. Let us first assume that the sources are uncorrelated. Then the asymptotic error can be shown to be (see Reddy \& Reddy 1996b)

$$
\Delta \theta_{i}^{2}=\frac{1}{L_{o}} \frac{\left[\operatorname{Re}\left(\alpha^{\mathrm{H}} \mathbf{R}_{n w} \Delta \mathbf{a}_{f}\left(\theta_{i}\right)\right)\right]^{2}}{\left[\mathbf{v}_{1}^{\mathrm{H}}\left(\theta_{i}\right) \mathbf{R}_{n w}^{\mathrm{H}} \mathbf{P}_{n} \mathbf{R}_{n w} \mathbf{v}_{f_{1}}\left(\theta_{i}\right)\right]^{2}},
$$

where

$$
\Delta \mathbf{a}_{f}\left(\theta_{i}\right)=\frac{1}{K} \sum_{l=1}^{K} \mathbf{Z}_{l}^{\mathrm{T}} \mathbf{J}_{\xi}^{-1} \Delta \mathbf{a}\left(\theta_{i}\right) \mathbf{e}^{-j(l-1) \theta_{i}},
$$

which we define as the effective error along the direction of the $i$ th source in the transformed steering vector due to the approximation. Note from (19) that the asymptotic error in the $i$ th DOA estimate is dependent only on the effective error vector $\Delta \mathbf{a}_{f}\left(\theta_{i}\right)$. The norm of this vector can be shown to decrease with spatial smoothing (see Appendix A of Reddy \& Reddy 1996b). Thus, we can expect the smoothing to improve the asymptotic performance of the Root-MUSIC applied to the transformed UCA data. Expression (19), however, holds good only when the sources are uncorrelated. For the case of correlated sources, simplification of (18) leads to lengthy expressions even for a two-source case, and hence, is not considered here.

\subsection{Forward-backward spatial smoothing}

Forward-backward spatial smoothing (William et al 1988) can also be extended to UCA by applying the transformation $\mathbf{J}_{\xi}^{-1}$ to the covariance matrix $\mathbf{R}^{t}$ (given in (6)) provided the term $\Delta \mathbf{R}$ is negligible. If $K$ is the number of virtual subarrays, then the forward-backward smoothed covariance matrix is given by

$$
\mathbf{R}_{f b}^{t}=\frac{1}{2 K} \sum_{l=1}^{K} \mathbf{Z}_{l}^{\mathrm{T}}\left[\mathbf{J}_{\xi}^{-1} \mathbf{R}^{t} \mathbf{J}_{\xi}^{-1}+\tilde{\mathbf{I}}\left(\mathbf{J}_{\xi}^{-1} \mathbf{R}^{t} \mathbf{J}_{\xi}^{-1}\right)^{*} \tilde{\mathbf{I}}\right] \mathbf{Z}_{l}
$$

where $\tilde{\mathbf{I}}$ is the exchange matrix and (.)* denotes complex conjugate of (.). The prewhitening matrix in this case is also given by $\mathbf{R}_{n w}$ (defined in $\S 3$ ) because $\tilde{\mathbf{I}}\left(\mathbf{J}_{\xi}^{-1} \mathbf{J}_{\xi}^{-1}\right)^{*} \tilde{I}=\mathbf{J}_{\xi}^{-1} \mathbf{J}_{\xi}^{-1}$. Note also from (4) that $\tilde{\mathbf{I}} \mathbf{A}^{*}=\mathbf{A}$. Hence, when the signal covariance matrix $\mathbf{S}$ is real (either the signals are uncorrelated or the correlation between the signals is real), it is easy to see that $\mathbf{R}_{f b}^{t}$ reduces to $\mathbf{R}_{f}^{t}$. This suggests that the forward-backward smoothing gives the 
same performance as the forward smoothing when $\mathbf{S}$ is real. When $\mathbf{S}$ is complex, it can be shown (Williams et al 1988; Pillai \& Kwon 1989) that the forward-backward smoothing can handle up to $\lfloor 2(2 \mathcal{D}+1) / 3\rfloor$ coherent signals in contrast to the forward smoothing which handles up to $\lfloor(2 \mathcal{D}+1) / 2\rfloor$ coherent signals only.

Taking into account the structure of $\mathbf{a}(\theta)$ given in (4), it can be shown that the effective correlation coefficient $\left(\rho_{f b}\right)$ between the sources after forward-backward smoothing, in the case of UCA, is given by (assuming $\Delta \mathbf{R}$ to be negligible)

$$
\left|\rho_{f b}\right|=|\rho|\left|\frac{\sin \left(K\left(\theta_{i}-\theta_{j}\right) / 2\right) \cos \psi}{K \sin \left(\left(\theta_{i}-\theta_{j}\right) / 2\right)}\right|,
$$

where $\rho=|\rho| e^{j \psi}$. Observe from (22) and (9) that the effective correlation with forwardbackward smoothing is same as that with forward smoothing only, when $\psi$ is zero, i.e., $\rho$ is real. When $\psi$ is an odd multiple of $\pi / 2$, the effective correlation with forward-backward smoothing reduces to zero for all $K$.

The mean square error (MSE) in the $i$ th DOA estimate that we obtain by applying the Root-MUSIC with forward-backward smoothing to the transformed UCA data, due to both the finite data perturbations and the error due to approximation (cf. (3)), can be shown to be (see Rao \& Hari 1990)

$$
\begin{aligned}
E\left[\Delta \theta_{i}^{2}\right]_{f b}= & \frac{1}{N K^{2} 2\left[\mathbf{v}_{f_{1}}^{\mathrm{H}}\left(\theta_{i}\right) \mathbf{R}_{n w}^{\mathrm{H}} \mathbf{P}_{n} \mathbf{R}_{n w} \mathbf{v}_{f_{\mathrm{l}}}\left(\theta_{i}\right)\right]^{2}} \\
& \quad \times \sum_{p=1}^{K} \sum_{q=1}^{K}\left[\left[\alpha^{\mathrm{H}} \mathbf{R}_{p q} \alpha \beta^{\mathrm{H}} \mathbf{R}_{q p} \beta+\left|\alpha^{\mathrm{H}} \mathbf{R}_{p q} \beta\right|^{2}\right]\right. \\
& \left.\quad+2\left[\operatorname{Re}\left(\alpha^{\mathrm{H}} \Delta \mathbf{R}_{\mathbf{F B}} \beta\right)\right]^{2}\right] . \\
\alpha= & \mathbf{P}_{n} \mathbf{R}_{n w} \mathbf{v}_{f_{\mathrm{l}}}\left(\theta_{i}\right) ; \quad \beta=\left(\mathbf{R}_{\mathbf{F B}}\right)_{s}^{\#} \mathbf{R}_{n w} \mathbf{v}_{f}\left(\theta_{i}\right), \\
\left(\mathbf{R}_{\mathbf{F B}}\right)_{s}= & \mathbf{R}_{n w} \mathbf{A}_{f} \mathbf{S}_{f b} \mathbf{A}_{f}^{\mathrm{H}} \mathbf{R}_{n w}^{\mathrm{H}} ; \quad \Delta \mathbf{R}_{\mathbf{F B}}=\frac{\Delta \mathbf{R}_{\mathbf{F}}+\tilde{\mathbf{I}}\left(\Delta \mathbf{R}_{\mathbf{F}}\right)^{*} \tilde{\mathbf{I}}}{\mathbf{2}},
\end{aligned}
$$

where $\mathbf{P}_{n}$ and $\mathbf{S}_{f b}$ are the noise projection matrix and the signal covariance matrix, respectively, obtained with the forward-backward smoothing. The first two terms in (23) are due to finite data perturbations and the third term is because of the error due to the approximation. The performance with forward-backward smoothing is the same as that with forward smoothing when $\mathbf{S}$ is real. Hence, the expression (19) can be used for the asymptotic performance of the Root-MUSIC with forward-backward smoothing also, when the sources are uncorrelated.

The analysis carried out, so far, assumes omni directional sensors. Spatial smoothing can also be extended to UCA with directional sensors (see Reddy \& Reddy 1998) and the analysis of Section 4 is applicable for UCA with directional elements.

\section{Numerical and simulation results}

In the simulations and numerical evaluation, a UCA with identical elements was considered. We considered two sources with equal powers in all the simulations. The snapshot 


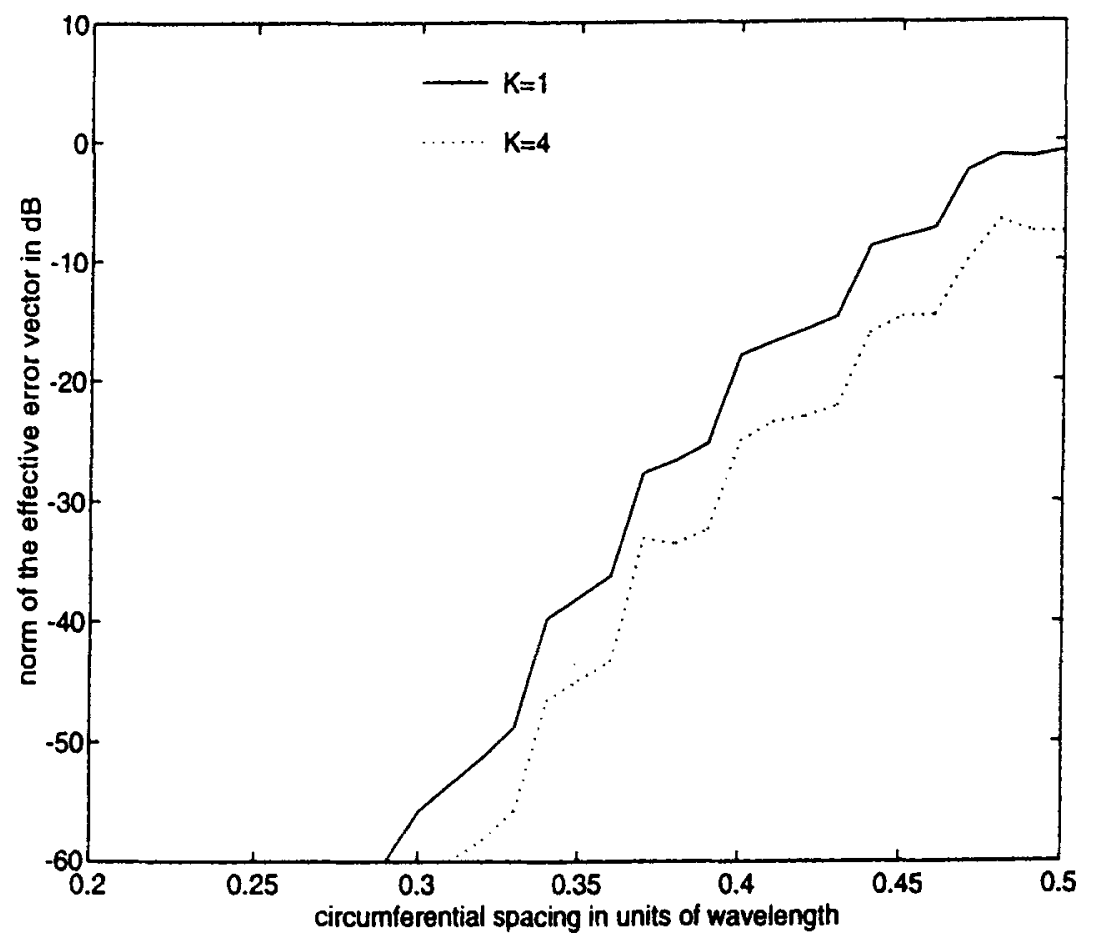

Figure 1. Norm of the effective error vector in the transformed steering vector of UCA as a function of circumferential spacint $(L=30)$.

vector was the sum of noise and signal vectors which were generated separately. The noise vector consisted of zero mean, unit variance, independent complex circularly Gaussian random variables. In the case of uncorrelated signals, the signal vector consisted of two zero mean, independent complex circularly Gaussian random variables of equal variance $\sigma_{s}^{2}$, where $\sigma_{s}^{2}$ was chosen to give the desired signal powers. In the case of correlated signals, the second signal $s_{2}(t)$ was generated as $s_{2}(t)=\rho s_{1}(t)+\sqrt{1-|\rho|^{2}} s(t)$ where $s_{1}(t)$ and $s(t)$ are zero mean, independent complex circularly Gaussian random variables with variance $\sigma_{s}^{2}$ and $\rho$ is the correlation coefficient between $s_{1}(t)$ and $s_{2}(t)$. The estimates of the DOA's were obtained by averaging over 100 Monte Carlo runs. The number of snapshots, the number of virtual subarrays, the total array size and the particulars of signal scenario are described in the captions of figures and tables. The SNR indicated in the figures refers to the value at the input of the sensor element. For spectral MUSIC, the search for DOA's was conducted in steps of $0.002^{\circ}$.

Figure 1 shows variation of the norm of the effective error vector in the transformed steering vector (see (20)). Note that as the spacing between the elements increases, the norm of the error vector increases and becomes quite large when the spacing approaches $\lambda / 2$. Also, the norm decreases with smoothing. This behaviour is in accordance with our predictions.

Table 1 compares the performance of the spectral MUSIC applied to the UCA output with that of the Root-MUSIC (without smoothing) applied to the transformed data for various values of $d$ (circumferential spacing between the elements). We note the following from 
Table 1. Performance of the Root-MUSIC applied to transformed UCA data as a function of the circumferential spacing between the elements (without smoothing) $\left(L=30, N=100, \mathrm{DOA}^{\prime} \mathrm{s}=0^{\circ}\right.$ and $7^{\circ}$, $\mathrm{SNR}=3 \mathrm{~dB}, \rho=0.0$ ).

\begin{tabular}{lccc}
\hline $\begin{array}{l}\text { Circumferential } \\
\text { spacing between } \\
\text { the elements }\end{array}$ & \multicolumn{3}{c}{ MSE in DOA estimate $\left(\mathrm{deg}^{2}\right)$} \\
\cline { 2 - 4 }$(d)$ & $\begin{array}{c}\text { Spectral MUSIC applied } \\
\text { to UCA data }\end{array}$ & \multicolumn{2}{c}{$\begin{array}{c}\text { Root-MUSIC applied to } \\
\text { transformed UCA data }\end{array}$} \\
\hline $0.32 \lambda$ & 0.2275 & 0.07972 & Evaluation of $(14)$ \\
$0.34 \lambda$ & 0.1570 & 0.06487 & 0.08477 \\
$0.36 \lambda$ & 0.1104 & 0.06454 & 0.05793 \\
$0.38 \lambda$ & 0.07045 & 0.04952 & 0.05382 \\
$0.40 \lambda$ & 0.04468 & 0.03284 & 0.03762 \\
$0.42 \lambda$ & 0.03259 & 0.03140 & 0.02803 \\
$0.44 \lambda$ & 0.02222 & 0.04897 & 0.02736 \\
$0.46 \lambda$ & 0.01627 & 0.08968 & 0.03515 \\
$0.48 \lambda$ & 0.01116 & 0.30931 & 0.07188 \\
\hline
\end{tabular}

the results. The performance of the spectral MUSIC improves as the spacing increases. This is because of the increased aperture that we get with increasing value of $d$. The performance of the Root-MUSIC is better than that of the spectral MUSIC when the spacing between the elements is less than $0.42 \lambda$. But, as the spacing is increased further, the Root-MUSIC performance degrades and becomes worse as $d$ approaches $\lambda / 2$. This is because, at larger spacings, the error in the transformed steering vector is quite large (see figure 1). The simulation results agree reasonably well with the theoretical values obtained from the numerical evaluation of (14) when $d$ is less than $0.46 \lambda$. For larger values of $d$, however, the difference between the two increases since the theoretical expression (14) is less accurate when the term $\Delta \mathbf{R}_{F}$ becomes large.

To see if the smoothing reduces the effect of the error introduced due to the approximation (cf. (3)) in the case of both uncorrelated and correlated scenarios, and to evaluate the utility of the theoretical result (18) and (19), we applied the Root-MUSIC with forward smoothing to the covariance matrix $\left(\mathbf{R}_{f}^{t}\right)_{w}$. The result so obtained from this is referred to as the asymptotic performance from the algorithm. Table 2 gives this result along with the theoretical values evaluated from (18) and (19) for various values of subarrays. Note from the results of table 2 that the MSE is maximum for $K=1$ (no smoothing) and it drops significantly with smoothing. Consider the results shown in the table for uncorrealted source scenario. As the smoothing is increased beyond $K=8$, the performance starts deteriorating because of the reduction in the aperture. The results predicted from (19) are not identical to those obtained from the algorithm since the theoretical expressions are accurate for small values of errors and $\Delta \mathbf{a}(\theta)$, the error in the present case, is not small as $d$ is close to $\lambda / 2$. The small fluctuations in the values with $K$ are because of the fact that the rate at which the norm of the effective error vector (cf. (20) ) and the effective correlation (with smoothing) fall varies with $K$. In fact, the fall in the effective correlation with $K$ is not monotonic (cf. (9)); it is rather oscillatory, tending to zero only as $K$ tends to infinity. This explains why the performance for $K=7$ is inferior to that for $K=5$ and 6 . Since the forward-backward smoothing gives the same performance as the forward smoothing when $S$ is real, these results also hold for the forward-backward smoothing case.The results for 
Table 2. Asymptotic performance of the Root-MUSIC with forward smoothing applied to transformed UCA data for two-source case. $\left(L=30, d=0.46 \lambda, \mathrm{DOA}^{\prime} \mathrm{s}=0^{\circ}\right.$ and $\left.7^{\circ}, \mathrm{SNR}=3 \mathrm{~dB}\right)$

\begin{tabular}{lcccc}
\hline $\begin{array}{l}\text { Number of } \\
\text { virtual } \\
\text { subarrays, } \\
(K)\end{array}$ & \multicolumn{4}{c}{$\begin{array}{c}\text { Asymptotic performance of the Root-MUSIC } \\
\text { with forward smoothing }\left(\Delta \theta_{i}^{2} \text { in } \mathrm{deg}^{2}\right)\end{array}$} \\
\cline { 2 - 5 } \cline { 2 - 5 } & \multicolumn{3}{c}{$\rho=0.0$} & \multicolumn{2}{c}{$\rho=0.9$} \\
\hline 1 & $0.4852 \times 10^{-1}$ & $0.5359 \times 10^{-1}$ & $0.4852 \times 10^{-1}$ & $0.4893 \times 10^{-1}$ \\
2 & $0.1110 \times 10^{-1}$ & $0.1148 \times 10^{-1}$ & $0.2929 \times 10^{-1}$ & $0.3105 \times 10^{-1}$ \\
3 & $0.2002 \times 10^{-1}$ & $0.2018 \times 10^{-1}$ & $0.4686 \times 10^{-1}$ & $0.4791 \times 10^{-1}$ \\
4 & $0.4491 \times 10^{-2}$ & $0.4684 \times 10^{-2}$ & $0.2819 \times 10^{-1}$ & $0.2951 \times 10^{-1}$ \\
5 & $0.3120 \times 10^{-3}$ & $0.3594 \times 10^{-3}$ & $0.7611 \times 10^{-2}$ & $0.8719 \times 10^{-2}$ \\
6 & $0.5329 \times 10^{-3}$ & $0.5958 \times 10^{-3}$ & $0.9496 \times 10^{-2}$ & $0.1082 \times 10^{-1}$ \\
7 & $0.2543 \times 10^{-2}$ & $0.2624 \times 10^{-2}$ & $0.2612 \times 10^{-1}$ & $0.2737 \times 10^{-1}$ \\
8 & $0.1255 \times 10^{-3}$ & $0.1524 \times 10^{-3}$ & $0.5241 \times 10^{-2}$ & $0.6161 \times 10^{-2}$ \\
9 & $0.2663 \times 10^{-3}$ & $0.3085 \times 10^{-3}$ & $0.7199 \times 10^{-2}$ & $0.8350 \times 10^{-2}$ \\
10 & $0.3612 \times 10^{-3}$ & $0.4167 \times 10^{-3}$ & $0.8421 \times 10^{-2}$ & $0.9799 \times 10^{-2}$ \\
11 & $0.5708 \times 10^{-3}$ & $0.6524 \times 10^{-3}$ & $0.1081 \times 10^{-1}$ & $0.1257 \times 10^{-1}$ \\
12 & $0.1155 \times 10^{-2}$ & $0.1289 \times 10^{-2}$ & $0.1653 \times 10^{-1}$ & $0.1890 \times 10^{-1}$ \\
\hline
\end{tabular}

the case of correlated sources are also given in table 2 . Since the correlation coefficient is real, these results hold for both the forward and forward-backward smoothing. Smoothing improves the performance in this case too. However, the improvement is not as much as with uncorrelated sources.

To see the differential impact of forward and forward-backward smoothing in the presence of highly correlated and closely spaced sources with finite data, we considered a scenario with $\rho=0.95 e^{j \pi / 4}$ and $N$ (number of snapshots) $=100$, keeping the source spacing nearly equal to the beamwidth of the UCA, and evaluated the MSE as a function of $K$. Table 3 gives the simulation results and the theoretical values predicted by (14) and (23). Note that the forward-backward smoothing yields much superior performance as compared

Table 3. Finite data performance of the Root-MUSIC with smoothing applied to transformed UCA data with omni directional elements for correlated sources. $\left(L=50, d=0.34 \lambda, N=100, \mathrm{DOA}^{\prime} \mathrm{s}=0^{\circ}\right.$ and $10^{\circ}, \rho=0.95 e^{j \pi / 4}, \mathrm{SNR}=3 \mathrm{~dB}$ )

\begin{tabular}{|c|c|c|c|c|c|}
\hline \multirow{4}{*}{$\begin{array}{l}\text { Number of } \\
\text { virtual } \\
\text { subarrays } \\
(K)\end{array}$} & \multicolumn{5}{|c|}{ MSE in DOA estimate $\left(\mathrm{deg}^{2}\right)$} \\
\hline & \multirow{3}{*}{$\begin{array}{l}\text { Spectral MUSIC } \\
\text { applied to } \\
\text { UCA data }\end{array}$} & \multicolumn{4}{|c|}{ Root-MUSIC with smoothing applied to transformed data } \\
\hline & & \multicolumn{2}{|c|}{ Forward-Backward smoothing } & \multicolumn{2}{|c|}{$\begin{array}{ll}\text { Forward smoothing } \\
\end{array}$} \\
\hline & & Simulation & Evaluation of (23) & Simulation & Evaluation of (14) \\
\hline 1 & 0.02485 & 0.003313 & 0.003058 & 0.01942 & 0.01880 \\
\hline 2 & & 0.003419 & 0.003223 & 0.01737 & 0.01814 \\
\hline 3 & & 0.003568 & 0.003449 & 0.01713 & 0.01784 \\
\hline 4 & & 0.004373 & 0.004053 & 0.01694 & 0.01867 \\
\hline 5 & & 0.007673 & 0.007213 & 0.02635 & 0.02915 \\
\hline 6 & & 0.01883 & 0.01789 & 0.05958 & 0.06443 \\
\hline 7 & & 0.01569 & 0.01582 & 0.04390 & 0.04929 \\
\hline 8 & & 0.01462 & 0.01556 & 0.03724 & 0.04505 \\
\hline 9 & & 0.01817 & 0.02131 & 0.05248 & 0.06151 \\
\hline 10 & & 0.01622 & 0.02016 & 0.05238 & 0.05833 \\
\hline
\end{tabular}


to the forward smoothing only. Further, the Root-MUSIC with forward-backward smoothing applied to the transformed UCA data performs better than the spectral MUSIC applied to the UCA data for all values of $K$ (and much better at lower values of $K$ ). When $K$ increases, the aperture comes down and the performance will start degrading when the aperture effect becomes predominant. The difference between the simulation results and the predicted values (particularly in the forward smoothing case) can be attributed to the fact that the smoothing reduces the effect of finite data perturbations in addition to reducing the correlation among the sources, and hence, the actual MSE will be less than the value given by (14) (see Reddy \& Reddy 1996a for discussion on this issue).

\section{Conclusions}

This paper extends the spatial smoothing to UCA and analyzes the DOA estimation performance of the Root-MUSIC with smoothing applied to the transformed UCA data. It is shown that the smoothing helps in reducing the effect of the errors that arise while extending the Root-MUSIC to UCA, in addition to reducing the correlation among the sources and the effect of noise perturbations due to finite data.

\section{References}

Davies D E N 1983 The handbook of antenna design (London: Peter Peregrinus) vol. 2, chap. 12 Mathews C P, Zoltowski M D 1994 Eigenstructure techniques for 2-D angle estimation with uniform circular arrays. IEEE Trans. Signal Process 42: 2395-2407

Pillai S U, Kwon B H 1989 Forward/backward spatial smoothing techniques for coherent signal identification. IEEE Trans. Acoust. Speech Signal Process. 37: 8-15

Rao B D, Hari K V S 1990 Effect of spatial smoothing on the performance of MUSIC and minimum-norm method. Inst. Elec. Eng. Proc. 137: 449-458

Reddy K M, Reddy V U 1996a Further results in spatial smoothing. Signal Process. 48: 217-224

Reddy K M, Reddy V U 1996b Analysis of interpolated arrays with spatial smoothing. Signal Process. 54: 261-272

Reddy K M, Reddy V U 1998 Analysis of spatial smoothing with uniform circular arrays. IEEE Trans. Signal Process. (submitted)

Reddy V U, Paulraj A J, Kailath T 1987 Performance analysis of the optimum beamformer in the presence of correlated sources and its behaviour under spatial smoothing. IEEE Trans. Acoust. Speech Signal Process. 35: 927-936

Shan T J, Wax M, Kailath T 1985 On spatial smoothing for directions of arrival estimation of coherent signals. IEEE Trans. Acoust. Speech Signal Process. 33: 806-811

Tewfik A H, Hong W 1992 On the application of uniform linear array bearing estimation techniques to uniform circular arrays. IEEE Trans. Signal Process. 40: 1008-1011

Williams R T, Prasad S, Mahalanabis A K, Sibul L H 1988 An improved spatial smoothing technique for bearing estimation in a multipath environment. IEEE Trans. Acoust. Speech Signal Process. 36: 425-431 